\section{SEPSE: RESSUSCITAÇÃO HEMODINÂMICA}

Autoria: Associação de Medicina Intensiva Brasileira, Sociedade Brasileira de Infectologia

Elaboração final: 07 de julho de 2009

Participantes: Glauco Westphal, Eliezer Silva, Reinaldo Salomão, Flavia Machado, Wanderley Marques Bernardo, Instituto Latino Americano de Sepse

\section{Descrição do método de coleta de evidência}

A fonte primária de consulta foi a base de dados MEDLINE através de acesso ao serviço PubMed de Pesquisa Bibliográfica em Publicações Médicas. Pela interface MeSH (Medical Subject Heading), inseriu-se os descritores da seguinte forma: (severe sepsis OR septic shock AND early goal directed therapy), (severe sepsis OR septic shock AND early goal directed therapy OR central venous oxygen saturation OR venous oximetry), (severe sepsis OR septic shock OR critically ill AND right atrial pressure OR central venous pressure OR cvp AND pulmonary artery occlusion pressure OR POAP AND pulmonary artery catheter AND arterial pressure OR pulse pressure variation AND fluid responsiveness OR volume expansion OR fluid resuscitation OR cardiac preload), (severe sepsis OR septic shock OR critically ill AND fluid resuscitation OR crystalloids OR colloids OR albumin OR synthetic colloids), (severe sepsis OR septic shock AND ressuscitation AND vasopressors OR dopamine OR norepinephrine OR epinephrine OR vasopressine), (severe sepsis OR septic shock AND ressuscitation AND inotropics OR dobutamine, OR dopamine OR epinephrine OR Isoproterenol OR Milrinone OR Amrinone OR levosimendan), (severe sepsis OR septic shock OR critically ill AND hemodynamics AND bicarbonate OR bicarbonate therapy AND acidosis OR lactic acidosis), (severe sepsis OR septic shock OR critically ill AND volume expansion OR fluid resuscitation OR positive fluid balance OR negative fluid balance OR fluid balance OR fluid management OR fluid therapy). As fontes secundárias consultadas foram as bases de dados Cochrane, Ovid e Trip Database. As buscas foram direcionadas para atender perguntas estruturadas na metodologia P.I.C.O. (População, Intervenção, Comparação e Outcome ou Desfecho).

\section{Graus de recomendação e força de evidência}

A: Estudos experimentais ou observacionais de melhor consistência.

B. Estudos experimentais ou observacionais de menor consistência.

C. Relatos de casos (estudos não controlados)

D. Opinião desprovida de avaliação crítica, baseada em consensos, estudos fisiológicos ou modelos animais

\section{Introdução}

Pacientes com sepse grave e choque séptico apresentam vasodilatação e grandes perdas hídricas para o espaço intersticial que podem somar-se à depressão miocárdica. O consequente comprometimento do fluxo sanguíneo pode resultar em isquemia de extensos territórios que, se não revertida precocemente, precipita o desenvolvimento de disfunção de múltiplos órgãos elevando a chance de óbito ${ }^{1}(\mathbf{B})$. Na fase inicial do tratamento deve-se buscar a reversão precoce da hipóxia tecidual pela restauração do fluxo sanguíneo global (fluxo sanguíneo = débito cardíaco - DC), obtida com reposição volêmica agressiva e/ou uso de vasopressores e de inotrópicos. A escolha da opção terapêutica mais apropriada deve ser norteada por metas pré-determinadas, com ênfase nos marcadores de fluxo e de oxigenação tecidual. A atenção aos marcadores hemodinâmicos permite avaliar a resposta dos pacientes às medidas terapêuticas, ajustá-las de forma a obter o maior benefício, e evitar iatrogenias. Os questionamentos que se seguem buscam respostas devidamente embasadas a tópicos fundamentais da ressuscitação hemodinâmica do paciente com sepse grave e choque séptico.

\section{A ressuscitação hemodinâmica precoce guiada por metas está indicada em todos os pacientes com sepse grave?}

A "Terapia Precoce Guiada por Metas" é uma estratégia de ressuscitação hemodinâmica que busca atingir objetivos hemodinâmicos com a readequação da oferta de oxigênio aos tecidos antes que a disfunção de múltiplos órgãos se desenvolva ${ }^{2,3}(\mathbf{A})$. Esta estratégia está indicada em pacientes com sepse grave e lactato sérico superior a $4 \mathrm{mmol} / \mathrm{l}$, ou hipotensão refratária (PAS $<90 \mathrm{mmHg}$ ou PAM < $65 \mathrm{mmHg}$ ) à infusão hídrica inicial e precoce de 20 a $30 \mathrm{ml} / \mathrm{kg}$ de cristalóide, ou dose correspondente de colóide $^{2}(\mathbf{A})$. As seguintes metas devem ser alcançadas num prazo de 6 horas: pressão venosa central (PVC) entre 8-12 mmHg, pressão arterial média (PAM) $\geq 65 \mathrm{mmHg}$, débito urinário $\geq$ $0,5 \mathrm{ml} / \mathrm{kg} /$ hora e saturação venosa central de oxigênio (SvcO2) $\geq 70 \%$. Vários estudos demonstraram que a aplicação desta estratégia, neste contexto, está associada à redução signifi cativa do risco de morte ${ }^{2}(\mathbf{A})^{4}(\mathbf{B})^{5}(\mathbf{D})$. Originalmente, foi demonstrado redução do risco absoluto de morte em $16 \%(\text { NNT }=6)^{2}(\mathbf{A})$.

Recomendação: A "Terapia Precoce Guiada por Metas" está recomendada para pacientes sépticos graves que apresentem hipotensão refratária a volume e/ou lactato sérico elevado ( $\geq 4$ $\mathrm{mmol} / \mathrm{l})^{2}(\mathbf{A})$. Não há evidências que justifiquem seu uso quando hipotensão e hiperlactatemia estão ausentes ${ }^{2}(\mathbf{A})^{1,6}(\mathbf{B})$.

\section{Existe benefício em monitorar a saturação venosa central de oxigênio (SVCO2)?}

A SvcO2 < 60\% é sinalizador de baixo DC no infarto agudo do miocárdio ${ }^{7}(\mathbf{C})$, e associada a maior mortalidade quando presente à admissão na unidade de terapia intensiva $(U T I)^{8}(\mathbf{B})$. Em razão da má distribuição do fluxo sanguíneo e baixo aproveitamento tecidual de oxigênio, a SvcO2 pode estar elevada (> 70\%) em pacientes sépticos ${ }^{1}(\mathbf{B})$. No início da sepse, o baixo aproveitamento tecidual de oxigênio ao baixo DC relacionado à hipovolemia e/ou disfunção miocárdica (hipóxia isquêmica), e a SvcO2 resulta diminuídaํ'(B). Em 762 pacientes foi observado que os benefícios obtidos com base nos valores de oferta e consumo de oxigênio obtidos com a monitorização do DC foram similares aos obtidos com a terapia guiada pela saturação de oxigênio do sangue venoso misto $(\mathrm{SvO} 2)^{9}(\mathbf{A})$. Em estudo observacional que incluiu 36 pacientes graves, notouse que após a expansão hídrica e estabilização da PAM, 31 ainda apresentavam SvcO2 <65\% e lactato $>2 \mathrm{mmol} / \mathrm{l}$. Só a partir 
de oferta hídrica adicional houve normalização do lactato e da $\mathrm{SvcO}^{8}(\mathbf{B})$. A comparação de dois grupos de pacientes com sepse grave, com e sem orientação terapêutica pela SvcO2, demonstrou que os objetivos das variáveis mecânicas tradicionais (PAM, PVC e débito urinário) foram alcançados em ambos os grupos. No entanto, a SvcO2 foi signifi cativamente maior e a mortalidade significativamente menor no $\operatorname{Grupo}^{12}(\mathbf{A})$.

Recomendação: A restauração da estabilidade hemodinâmica baseada em variáveis mecânicas tradicionais como PAM, PVC e débito urinário não é suficiente para a restauração da oxigenação tecidual e resultar em benefício em termos de prognóstico $(\mathbf{A})^{8}(\mathbf{B})$. A orientação terapêutica baseada na $\mathrm{SvcO} 2$ e na sua normalização precoce resulta na recuperação do fluxo sanguíneo ${ }^{9}(\mathbf{A})$ e redução significativa da mortalidade ${ }^{2}(\mathbf{A})$. Assim, recomenda-se sua monitorização.

\section{Há vantagens prognósticas em monitorizar a svco2 após a fase de ressuscitação? por quanto tempo após o início da ressuscitação essas metas devem ser perseguidas?}

Em estudo clínico randomizado alcançou-se o objetivo terapêutico em $95 \%$ dos pacientes com sepse grave e choque séptico em que se buscou a restauração da SvcO2 > 70\% em 6 horas. No grupo controle, a SvcO2 não fez parte dos objetivos terapêuticos. Apenas 60\% destes pacientes alcançaram Svc02 > 70\% ( $p<$ 0,001 ). Em ambos os grupos utilizaram-se também as seguintes metas: $P V C \geq 8 \mathrm{cmH} 20$, PAM $\geq 65 \mathrm{mmHg}$ e Débito Urinário $\geq$ $0,5 \mathrm{ml} / \mathrm{kg} /$ hora. Atingir a meta de $\mathrm{SvcO} 2>70 \%$ em 6 horas esteve associada a reduções signifi cativas da mortalidade hospitalar ( $p$ $=0,009)$, do tempo de ventilação mecânica $(p=0,001)$ e da permanência hospitalar $(p=0,001)^{2}(\mathbf{A})$. Vários outros estudos de controle têm corroborado estes benefícios5(D). Entretanto, não está claro que exista benefício em se objetivar manter uma SvcO2 acima de $70 \%$ após a fase inicial da ressuscitação ( 6 primeiras horas). É possível que o benefício alcançado se estenda pelas primeiras 24 horas, embora não haja evidências que suportem essa conduta.

Recomendação: Atingir precocemente a meta terapêutica de SvcO2 > 70\% tem impacto sobre a mortalidade, portanto deve ser cumprida o mais precocemente possível, preferencialmente nas 6 primeiras horas de tratamento ${ }^{2}(\mathbf{A})$. É possível que exista benefício na manutenção desses parâmetros otimizados durante as primeiras 24 horas de ressuscitação.

\section{0 uso de parâmetros para avaliar a adequação da reposição volêmica (PVC, POAP, variáveis dinâmicas) têm utilidade na prática clínica diária?}

Durante a fase de ressuscitação do choque, a avaliação mais adequada da reposição volêmica se dá pela aferição da responsividade cardiovascular (RC) à infusão hídrica. A avaliação da $\mathrm{RC}$ auxilia na diferenciação entre a necessidade de intensificar a expansão volêmica e a indicação de inotrópicos para reversão da hipóxia tissular.

4a. Avaliação estática da responsividade cardiovascular pressão venosa central (PVC) e pressão de oclusão da artéria pulmonar (POAP): Embora as pressões de enchimento ventricular figurem como métodos preferenciais de avaliação da RC, evidências recentes enfatizam a baixa sensibilidade e especificidade da PVC e da POAP para este fim ${ }^{10-12}(\mathbf{B})^{13}(\mathbf{C})$. Em estudo prospectivo que envolveu 44 voluntários saudáveis, foi observado que tanto o valor inicial quanto a variação da PVC e da POAP após infusão de volume não foram capazes de predizer a $\mathrm{RC}$ a volume ${ }^{10}(\mathbf{B})$. A análise retrospectiva de 96 pacientes sépticos demonstrou que a PVC $<8 \mathrm{mmHg}$ e a POAP $<12 \mathrm{mmHg}$ não foram capazes de predizer a responsividade a volume, com razão de verossimilhança positiva de 1,34 e 1,57 , respectivamente. O uso combinado das pressões de enchimento também não melhorou a acurácia das variáveis ${ }^{12}(\mathbf{B})$. Pacientes com sepse grave ou choque séptico foram estudados utilizando-se a PVC entre 8 e $12 \mathrm{mmHg}$ (associada à PAM, débito urinário e SvcO2) como um dos objetivos da ressuscitação hemodinâmica precoce. No grupo controle (sem aferição da Svc02), a média da PVC ao final das 6 horas foi $11,8 \pm 6,8 \mathrm{mmHg}$, enquanto no grupo tratamento (com Svc02) a média da PVC foi 13,8 \pm 4,4 mmHg. A PVC ao final das primeiras 6 horas esteve acima ou abaixo do objetivo inicial na maior parte dos pacientes. A PVC no grupo controle (com maior taxa de mortalidade) apresentou a média da PVC mais próxima do objetivo inicial, se comparada à média do grupo tratamento. Deste modo, utilizar valores de PVC entre 8-12 mmHg como meta exclusiva durante a ressuscitação hemodinâmica inicial em pacientes com sepse grave ou choque séptico pode produzir dano, principalmente se a ressuscitação volêmica for interrompida em pacientes responsivos com PVC $>8 \mathrm{~mm} \mathrm{Hg}$, mas que ainda não tenham alcançado a principal meta terapêutica: $\mathrm{SvcO} 2 \geq$ $70 \%{ }^{2}(\mathbf{A})^{14}(\mathbf{D})$. Em pacientes sépticos foi observado que PVC e POAP não foram capazes de discriminar indivíduos responsivos dos não responsivos a volume (área sob a curva $\mathrm{ROC}$ : $\mathrm{PVC}=0,51$ $\pm 0,12 ; \mathrm{POAP}=0,40 \pm 0,09)^{11}(\mathbf{B})$. Outros estudos semelhantes observaram os mesmo achados ${ }^{15-18}(\mathbf{B})$.

Recomendação: Os valores médios da PVC e da POAP, bem como a variação destas pressões após prova de volume não discriminam indivíduos responsivos dos não responsivos ${ }^{10-12,14-}$ ${ }^{18}$ (B). A PVC deve ser associada a outros parâmetros clínicos como PAM, débito urinário e SvcO2 para subsidiar a terapêutica²(A). Entretanto, em locais onde os métodos dinâmicos não estejam disponíveis podese basear a ressucitação hemodinâmica na PVC, pois sua otimização garantiria o recebimento de quantidade minimamente suficiente de volume.

4b. Avaliação dinâmica da responsividade cardiovascular - variação respiratória da pressão arterial $(\triangle P P)$ e variação respiratória da pvc ( $\triangle \mathrm{PVC})$ : Na análise do traçado da pressão arterial em 40 pacientes sépticos sob ventilação mecânica, constatou-se que a variação respiratória da pressão de pulso arterial $(\triangle \mathrm{Pp})$ tem alta sensibilidade $(94 \%)$ e especificidade $(96 \%)$ na identificação de indivíduos responsivos $(\triangle \mathrm{Pp}>13 \%)$ e não responsivos ( $\triangle \mathrm{Pp}<13 \%$ ), conferindo razão de verossimilhança positiva de 23,511(B). Estes dados foram corroborados em estudos prospectivos subsequentes ${ }^{15,16}(\mathbf{B})$. O método é validado para pacientes sob ventilação mecânica controlada, volume corrente entre 8 e 12 ml/kg e com ritmo sinusal ${ }^{11}(\mathbf{B})$. Na avaliação de 23 pacientes sépticos, observou-se que a diferença de amplitude 
da onda pletismográfica ( $\triangle$ Pplet) reflete o comportamento de $\triangle \mathrm{Pp}$ e é capaz de diferenciar responsivos de não responsivos com sensibilidade de 94\%, especificidade de $80 \%$ e razão de verossimilhança positiva de 4,7 (área sob a curva $\mathrm{ROC}=$ $0,94)^{17}(\mathbf{B})$. Dois outros estudos corroboram estes achados ${ }^{18,19}(\mathbf{B})$. A variação respiratória da PVC $(\triangle P V C)$ como preditor da $R C$ foi estudada em 33 pacientes ${ }^{20}(\mathbf{B})$. Foram incluídos pacientes em ventilação espontânea (36\%) e sob ventilação mecânica (64\%), nestes, a $\triangle \mathrm{PVC}$ foi aferida durante breve desconexão do ventilador mecânico. A queda inspiratória de $1 \mathrm{mmHg}$ na PVC demonstrou sensibilidade de $84 \%$, especifi cidade de $94 \%$ e razão de verossimilhança positiva de 14, para detecção de pacientes responsivos à expansão hídrica. Foram excluídos os pacientes que não gerassem esforço inspiratório suficiente para reduzir a POAP em $2 \mathrm{mmHg}$. Portanto, na vida real e na ausência da verificação da POAP, deve-se considerar a possibilidade de falso negativo quando $\triangle P V C<1 \mathrm{mmHg}$ é encontrado ${ }^{20,21}(\mathbf{B})$. Foram ainda estudados 21 pacientes, onde se observou que a $\triangle P V C$ não foi capaz de predizer a RC. Nove pacientes foram ventilados em pressão de suporte e em quatro pacientes não houve redução inspiratória de $2 \mathrm{mmHg}$ na $\operatorname{POAP}^{22}(\mathbf{C})$.

Recomendação: A $\triangle P p$ é um método simples, sensível e específico para avaliação da RC em pacientes com instabilidade hemodinâmica e sob ventilação mecânica controlada ${ }^{11,15,16}$ (B). A $\triangle$ Pplet é uma alternativa não invasiva à $\Delta \operatorname{pp}^{17,18}(\mathbf{B})$. A variação inspiratória da PVC, apesar de sensível e específica na identificação da RC, tem sua aplicabilidade limitada pela necessidade de aferição da variação concomitante POAP para evitar falso-negativos ${ }^{21}(\mathbf{B})$.

7. $\mathrm{Na}$ reposição volêmica, existe benefício em termos da evolução clínica com a utilização de soluções colóides (naturais ou sintéticos) em detrimento dos cristalóides? existe benefício no uso da albumina em alguma subpopulação específica?

Em revisão sistemática incluindo 30 ensaios clínicos randomizados, totalizando 1419 pacientes, comparou-se o uso da albumina humana com cristalóides em pacientes graves e hipovolêmicos, grandes queimados ou hipoalbuminêmicos. Concluiu-se que a o uso da albumina humana estava associada ao aumento de $6 \%$ no risco de morte ${ }^{23}(\mathbf{B})$. Outra revisão sistemática analisou 37 ensaios clínicos randomizados. Vinte e seis compararam colóides com cristalóides ( $n=1622$ ). A ressuscitação volêmica com colóides esteve associada com aumento de $4 \%$ no risco absoluto de morte, sem que se observassem resultados diferentes em subgrupos de pacientes de diferentes patologias que requeiram ressuscitação volêmica ${ }^{24}(\mathbf{B})$. Em revisão sistemática mais recente, foram identificados 63 estudos, dos quais 55 apresentaram dados relacionados à mortalidade. Vinte e três compararam cristalóide com albumina humana, 16 compararam cristalóide com infusão de hidroxietil starch, 11 compararam cristalóide com gelatina modifi cada, nove compararam cristalóide com dextran e oito compararam dextran em cristalóide hipertônico com cristalóide isotônico. Não houve diferença na mortalidade após 28 dias de seguimento, permanência em ventilação mecânica, na UTI e hospitalar, bem como na duração de terapia renal substitutiva e no número de disfunções orgânicas ${ }^{25}(\mathbf{B}) .0$ estudo SAFE, um ensaio clínico randomizado realizado em 16 hospitais da Oceania, incluiu 6997 pacientes que necessitaram de ressuscitação hídrica por depleção volêmica. Compararamse os efeitos da ressuscitação hídrica realizada com albumina $4 \%$ e com solução salina sobre a mortalidade. Não houve diferença na mortalidade após o $28^{\circ} \mathrm{dia}$, permanência em ventilação mecânica, na UTI e hospitalar, bem como na duração de terapia renal substitutiva e no número de disfunções orgânicas ${ }^{26}(\mathbf{B})$. Na análise de subgrupos do estudo SAFE, observou-se mortalidade ao $28^{\circ}$ dia significativamente maior $(p=0,009)$ entre pacientes vítimas de trauma crânioencefálico (TCE) grave que receberam colóide. Esta diferença se confirmou em avaliação post hoc em que se analisou a mortalidade em um $\operatorname{ano}^{27}(\mathbf{B})$. Entre pacientes sépticos houve uma tendência a menor mortalidade entre aqueles que receberam colóide $(p=0,09)^{26}(\mathbf{B})$. Não se observou benefícios da infusão de albumina nos indivíduos com albumina basal $\leq 25 \mathrm{~g} / \mathrm{l}^{28}(\mathbf{B})$. Novos estudos são necessários para deixar mais claro o papel dos colóides nesses subgrupos.

Recomendação: Não há benefícios na utilização do uso de colóides como expansores plasmáticos durante a ressuscitação volêmica em pacientes graves. Pacientes sépticos também não parecem se beneficiar de seu uso ${ }^{26}(\mathbf{B})$. Não há, até o presente momento, subpopulação específica que se beneficie da infusão de $\operatorname{colóides}^{26-28}(\mathbf{B})$.

\section{Existe um vasopressor ideal para ser utilizado no paciente séptico?}

Ainfusão dedrogasvasopressoras deveseriniciada em pacientes sépticos sempre que a expansão volêmica não for suficiente para restaurar a pressão arterial e a perfusão orgânica ${ }^{4}(\mathbf{B})^{29}(\mathbf{D})$. Os efeitos dos vasopressores têm sido avaliados em vários estudos não cegos. Observou-se que tanto a dopamina quanto a noradrenalina são capazes de produzir elevações consistentes na PAM de pacientes sépticos. No entanto, a noradrenalina é mais potente que a dopamina e provavelmente mais efetiva na reversão do choque séptico ${ }^{30,31}(\mathbf{A})^{32-34}(\mathbf{B})^{35}(\mathbf{D})$. Em estudo prospectivo e randomizado que envolveu 32 pacientes sépticos, comparou-se dopamina à noradrenalina. 0 choque séptico foi revertido em $31 \%$ dos pacientes em que se infundiu dopamina contra $93 \%$ dos que receberam noradrenalina ${ }^{30}(\mathbf{A})$. Os resultados dos estudos que avaliaram o efeito dos vasopressores sobre a perfusão esplâncnica são mistos ${ }^{32}(\mathbf{B})^{36,37}(\mathbf{A})$. Comparando-se os efeitos da dopamina, noradrenalina e adrenalina em pacientes com choque séptico, os efeitos da dopamina e da noradrenalina sobre a circulação esplâncnica são similares ${ }^{38}(\mathbf{B})$. Em indivíduos hipovolêmicos, a vasoconstricção é deletéria para a função renal ${ }^{29}(\mathbf{D})$. Um ensaio clínico randomizado analisou 328 pacientes graves que apresentaram disfunção renal aguda para testar a hipótese de proteção renal proporcionada pela administração de baixas doses de dopamina. Não houve qualquer diferença entre nível de creatinina, necessidade de diálise, débito urinário e tempo de recuperação da função renal ${ }^{39}(\mathbf{A})$. Outros estudos sustentam a hipótese de que a noradrenalina tende a otimizar o fluxo sanguíneo renal e a resistência vascular renal em pacientes com choque séptico que tenham recebido expansão volêmica adequada ${ }^{40,41}(\mathbf{B})^{42}(\mathbf{C})$. Um recente estudo observacional incluiu 1058 pacientes que apresentaram 
choque em algum momento da internação. Houve aumento significativo da mortalidade na UTI ( $p<0,02)$ e hospitalar $(p<0,01)$ entre pacientes que utilizaram dopamina quando comparados aos indivíduos que não utilizaram este fármaco. A mortalidade não aumentou entre pacientes que receberam noradrenalina ${ }^{43}(\mathbf{B})$. A infusão de adrenalina pode ser utilizada como alternativa em pacientes que não respondem à expansão volêmica ou infusão de outras catecolaminas. Este fármaco provoca evidente elevação da PAM em indivíduos não responsivos a dopamina ou noradrenalina. No entanto, a adrenalina reduz a perfusão esplâncnica e renal e provoca elevações dos níveis do lactato sérico ${ }^{31}(\mathbf{A})^{32,38,44}(\mathbf{B})$. A vasopressina é um hormônio normalmente liberado pela hipófise em resposta à hipovolemia e ao aumento da osmolaridade plasmática. Provavelmente pela depleção da reserva hipofisária. Esta liberação tende a ser menor ou até mesmo interrompida em pacientes com choque séptico. Um terço dos pacientes com choque séptico desenvolvem deficiência relativa de vasopressina ${ }^{45}(\mathbf{B})^{46}(\mathbf{C})^{29}(\mathbf{D})$. Pequenos estudos observacionais ${ }^{47,48}(\mathbf{B})$ e randomizados ${ }^{49,50}(\mathbf{B})$ vêm demonstrando que a administração de baixas doses $(0,01$ a 0,04 unidades/min) de vasopressina em pacientes com choque séptico refratário a catecolaminas resulta em incremento da pressão arterial e redução da infusão das catecolaminas. No entanto, a vasopressina causa má perfusão esplâncnica ${ }^{51,52}$ (B). Recentemente, um estudo duplo-cego e randomizado que incluiu 778 pacientes com choque séptico, comparou indivíduos que utilizaram vasopressina associada à noradrenalina com outros que utilizaram apenas noradrenalina. Não houve diferença na mortalidade global. Observou-se, no entanto, que os pacientes menos graves (5 to $14 \mu \mathrm{g}$ de noradrenalina no momento da inclusão) tiveram significativa redução da mortalidade no grupo vasopressina, ao contrário dos pacientes mais graves ( $>15 \mu \mathrm{g}$ de noradrenalina). Estes dados sugerem que a precocidade da infusão do vasopressor (e de outras terapias) é o fator decisivo, mais do que o agente vasopressor específico ${ }^{53}(\mathbf{A})$.

Recomendações: Tanto a dopamina quanto a noradrenalina (administrados por cateter central sempre que possível) são fármacos de primeira escolha em pacientes com choque séptico ${ }^{35}(\mathbf{B})$. No entanto, a noradrenalina é mais potente que a dopamina e provavelmente mais efetiva na reversão do choque séptico em determinados pacientes ${ }^{30,31}(\mathbf{A})^{34}(\mathbf{B}) ;$ A infusão de vasopressores deve ser precedida e/ou acompanhada de expansão volêmica adequada ${ }^{35}(\mathbf{B})$; Adrenalina não é fármaco de primeira escolha em pacientes com choque séptico ${ }^{35}(\mathbf{B})$. Pode se considerar o uso de adrenalina como droga alternativa em pacientes com choque séptico e hipotensão refratária a outros vasopressores ${ }^{31}(\mathbf{A})^{44}(\mathbf{B})$; Doses baixas de dopamina não devem ser utilizadas para proteção renal ${ }^{39}(\mathbf{A}) ; 0$ uso de baixas doses (0,01 a 0,04 unidades/min) de vasopressina em pacientes com choque refratário a expansão volêmica adequada e administração de catecolaminas resulta na recuperação da pressão arterial. Não é fármaco de primeira escolha ${ }^{51,52}(\mathbf{B})$. A associação de vasopressina com noradrenalina não traz benefícios sobre a mortalidade ${ }^{53}(\mathbf{A})$.

9. Existe um inotrópico ideal para ser utilizado no paciente séptico com sinais de disfunção miocárdica?
O quadro hemodinâmico da sepse é caracterizado pelo estado hiperdinâmico com pressão arterial baixa ou normal, e resistência vascular sistêmica baixa. Apesar do DC frequentemente ser normal em pacientes sépticos que receberam expansão volêmica adequada, vários autores demonstraram haver disfunção miocárdica (queda da fração de ejeção do ventrículo esquerdo, dilatação ventricular, baixa resposta contrátil à expansão volêmica) em boa parte destes pacientes ${ }^{4,35}(\mathbf{B})^{29}(\mathbf{D})$. Diante da manutenção de sinais de hipofluxo após a expansão volêmica adequada e administração de vasopressores, deve-se considerar o uso de inotrópicos para que se alcance precocemente as metas terapêuticas pré-estabelecidas em termos de $\mathrm{SvcO}^{2}(\mathbf{A})^{4}(\mathbf{B})^{29}(\mathbf{D})$. Estudo clínico incluiu 263 pacientes randomizados em dois grupos: 0 grupo tratamento, que utilizou a Terapia Precoce Guiada por Metas com monitorização da SvcO2 além da PAM, PVC e débito urinário, e o grupo controle, no qual a SvcO2 não foi levada em conta. Com o objetivo de normalizar a SvcO2 nas primeiras 6 horas de tratamento, o grupo tratamento recebeu maior expansão volêmica ( 5 vs 3,5 I; p < 0,001), mais transfusão de hemácias ( $p<0,001)$ e maior terapia inotrópica com dobutamina $(13,7$ vs $0,8 \%, p<0,001)$. A terapia inotrópica foi iniciada sempre que a meta de SvcO2 não era alcançada após a otimização da volemia e da massa eritrocitária. A mortalidade foi significativamente menor no grupo tratamento $(30,5 \text { vs } 46.5 \%, p<0,009)^{2}(\mathbf{A})$. Outros estudos demonstraram que para situações onde não há obtenção dos objetivos de PAM, DC e/ou oxigenação tecidual apenas com expansão hídrica e vasopressores (dopamina ou noradrenalina), a associação da dobutamina é uma boa estratégia para elevar o DC, a oxigenação tecidual e a $\operatorname{PAM}^{54,55}(\mathbf{A})$. No entanto, a supranormalização das variáveis hemodinâmicas não traz vantagens prognósticas quando comparada à obtenção da normalização do DC ou da saturação venosa mista de oxigênio $(\mathrm{SvO} 2)^{9}(\mathbf{A})$. A supranormalização do DC e da oferta de oxigênio com doses elevadas de dobutamina está associada a aumento significativo da mortalidade ${ }^{53}(\mathbf{A})$. A infusão de adrenalina, apesar de influenciar positivamente o débito cardíaco, por se tratar de potente inotrópico, está fortemente associada a prejuízo de perfusão regional ${ }^{31}(\mathbf{A})^{44,56}(\mathbf{B})$. Os inibidores da fosfodiesterase (amrinona e milrinona) são vasodilatadores de meia vida longa, que podem provocar hipotensão prolongada, exigindo associação de vasopressores. Pequenos estudos demonstraram seus efeitos positivos sobre o DC, porém, não é possível analisar (por razões amostrais) seus efeitos sobre o prognóstico ${ }^{57}(\mathbf{A})^{58,59}(\mathbf{B})$. Poucos estudos avaliaram o uso do isoproterenol na sepse e choque séptico. Embora promova elevações significativas do DC, pode provocar hipotensão e taquicardia resultando em isquemia cardíaca $^{60}(\mathbf{B})^{61}(\mathbf{C})$. O levosimendan é um sensibilizador de cálcio favorecendo o acoplamento actina-miosina e melhora a contratilidade cardíaca sem elevar o consumo de oxigênio do miócito. Além disso, abre os canais de K+ ATP dependentes, causando vasodilatação. Não há grandes estudos em pacientes sépticos que respaldem o seu uso. Dois ensaios clínicos (REVIVE II e SURVIVE) estudaram pacientes com insuficiência cardíaca descompensada e fração de ejeção $<35 \%$. Embora o primeiro ( $n$ $=600$ ) tenha demonstrado que o levosimendan está associado à melhora clínica e redução na permanência hospitalar, o segundo 
( $n=1327$ ) não observou benefícios do uso deste fármaco sobre a mortalidade, quando comparado à dobutamina ${ }^{62,63}(\mathbf{A})$.

Recomendações: A dobutamina é o inotrópico de escolha no paciente séptico com sinais de disfunção miocárdica ${ }^{54,55}(\mathbf{A})$. A terapia inotrópica com dobutamina está indicada quando a meta de $\mathrm{SvcO} 2>70 \%$ não for alcançada com a expansão volêmica adequada e, eventualmente, transfusão de hemáceas ${ }^{4,35}(\mathbf{B})$. Se houver hipotensão seu uso deve ser acompanhado de um vasopressor ${ }^{35}$ (B); 0 débito cardíaco não deve ser supra-normalizado ${ }^{53}(\mathbf{A})$.

\section{Pacientes com acidose metabólica grave de origem lática devem receber reposição de bicarbonato?}

Acidose metabólica por si só não é doença, trata-se de um sinal de grave desequilíbrio na homeostase. Pode ser classificada em acidose metabólica orgânica e acidose metabólica mineral. A acidose metabólica orgânica lática (lactato $>4 \mathrm{mMol} / \mathrm{L}$ ) é um marcador de gravidade quando presente em pacientes sépticos. Soluções de bicarbonato frequentemente são utilizadas visando à estabilização hemodinâmica e a redução da infusão de vasopressores. Há dois estudos clínicos, randomizados, prospectivos e cegos realizados em pacientes com acidose metabólica lática. Em ambos a administração de bicarbonato de sódio não modificou qualquer parâmetro hemodinâmico, a necessidade de uso de catecolaminas ou índices globais

de oxigenação tissular. Mesmo em faixas extremas de pH (6,9 a 7,2; média $=7,13)$, os resultados negativos persistiram ${ }^{64,65}(\mathbf{B})$. Do mesmo modo, em pacientes com cetoacidose diabética a infusão de bicarbonato de sódio não traz nenhum beneficio para a normalização do pH sérico, havendo maior necessidade de infusão de potássio ${ }^{66}(\mathbf{B})$. Há uma série de relatos experimentais a respeito do efeito protetor da acidose metabólica, quando a hipóxia tissular está presente. Células de diversos tecidos submetidas à privação de oxigênio e incubadas com pH entre 6,5 e 7 sobrevivem por várias horas. As mesmas células igualmente privadas de oxigênio, mas incubadas com pH de 7,4 morrem em menos de 1 hora. Durante a acidose há diminuição global no metabolismo celular, através da interferência do $[\mathrm{H}+]$ que modificam a conformação espacial das enzimas celulares ${ }^{67-69}(\mathbf{D})$.

Recomendação: Não se recomenda a infusão de bicarbonato de sódio em pacientes com acidose orgânica lática e instabilidade hemodinâmica ${ }^{64,65}(\mathbf{B})$.

\section{Existe implicação prognostica com excesso de infusão Hídrica e balanço hídrico positivo?}

Pacientes com sepse grave e choque séptico apresentam grandes déficits de volume intravascular em decorrência de volumosas perdas hídricas para o espaço intersticial e de importante redução da capacitância venosa. A restauração do débito cardíaco e da perfusão tecidual depende de expansão rápida e agressiva do volume ${ }^{2,3}(\mathbf{A})^{1,4}(\mathbf{B})$. Em sendo a restauração do fluxo sanguíneo e re-oxigenação tecidual a tônica de ressuscitação volêmica, a relação entre ganhos e perdas de líquidos não tem utilidade durante a ressuscitação precoce para definir a necessidade de líquidos ${ }^{3}(\mathbf{A})$. A infusão hídrica deve ser norteada por metas clínicas pré-determinadas, com ênfase nos marcadores de oxigenação tecidual e de função orgânica ${ }^{1,4}(\mathbf{B})^{29}$ (D). A efetividade da "Terapia Precoce Guiada por Metas" foi testada em pacientes com sepse grave e choque séptico. No grupo tratamento (terapia orientada pela ScvO2), observou-se que a oferta hídrica nas primeiras 6 horas foi significativamente maior que do grupo controle $(5,0$ l vs 3,5 I; p < 0,001), resultando em maior Scv02. Entre a $7^{\mathrm{a}}$ e a $72^{\mathrm{a}}$ hora, o grupo controle necessitou significativamente de mais líquido $(p=0,01)$. Este acréscimo hídrico tardio não aumentou a $\mathrm{SvcO} 2$ em relação ao grupo tratamento $(p<$ 0,001 ), não reduziu o escore de disfunções orgânicas MODS ( $p<$ 0,001 ) e não impactou na mortalidade que foi significativamente maior $(p=0,009)^{2}(\mathbf{A})$. A otimização supra fisiológica do débito cardíaco e da oferta de oxigênio em populações heterogêneas de pacientes graves, não evidenciou benefícios em extrapolar os níveis fisiológicos das metas pré-definidas ${ }^{9,70}(\mathbf{A})$. Portanto, não há evidências de que pacientes responsivos a provas hídricas, que apresentam aumentos discretos do débito cardíaco ou variáveis de oxigenação normalizados, sejam beneficiados por cargas adicionais de volume ${ }^{71}(\mathbf{B})$. A avaliação prospectiva de 29 pacientes sépticos que desenvolveram disfunção renal demonstrou que a infusão contínua significativamente maior de líquidos (2037 +/- 1681 vs. $1116+/-1220 \mathrm{~mL}, \mathrm{p}<0,03)$ não resultou em melhora da função renal e esteve associada à piora significativa da oxigenação $(p<0,04)^{72}($ B $)$. O estudo SOAP (multicêntrico internacional de caráter observacional) arrolou 393 pacientes que desenvolveram lesão pulmonar aguda (LPA)/ síndrome de desconforto respiratório agudo (SDRA). O excesso de líquidos foi identificado como fator independente que influenciou a mortalidade. Os não sobreviventes tiveram balanço hídrico significativamente maior que os sobreviventes $(p<0,001)^{73}(\mathbf{B})$. Um estudo randomizado recente comparou as estratégias, liberal e restritiva de administração de líquidos em 1000 pacientes com LPA, dos quais $71 \%$ apresentavam pneumonia ou sepse como causa primária da lesão pulmonar. 0 balanço hídrico acumulado ao final das primeiras 72 horas foi de $5.100 \mathrm{ml}$ no grupo da estratégia liberal e $400 \mathrm{ml}$ no grupo da estratégia restritiva. Não houve diferença na mortalidade, no entanto houve redução significativa do tempo de ventilação mecânica $(p<0,001)$ e da permanência na UTI $(p<0,001)$ no grupo da estratégia restritiva ${ }^{74}(\mathbf{A})$. Na análise retrospectiva de 36 pacientes com choque séptico, observou-se que todos os 11 pacientes em que se obteve balanço hídrico negativo de $500 \mathrm{ml}$ em pelo menos 1 dos 3 primeiros dias de tratamento sobreviveram. Por outro lado, 20 dos 25 pacientes em que não foi possível tornar o balanço hídrico negativo morreram $(p<0,00001)$. Isso sugere que a obtenção de balanço hídrico negativo em 1 dos 3 primeiros dias de tratamento é preditor de sobrevivência em pacientes com choque séptico ${ }^{75}(\mathbf{B})$. Do total de pacientes arrolados no estudo SOAP, 1177 tinham diagnóstico de sepse. Observou-se que o balanço hídrico acumulado nas primeiras 72 horas do diagnóstico da sepse (OR; 1,1 por litro adicional; $95 \%$ IC, 1,1-1,1; $p$ $<0,001)$ e o balanço hídrico diário (OR; 1,8 por litro adicional; $95 \%$ IC $, 1,6-2,0 ; p<0,001$ ) foram preditores independentes de mortalidade. Porém, trata-se de pacientes mais graves, com maior número de órgãos disfuncionantes $(p<0,001)$ e maior valor médio do SOFA $(p<0,001)^{76}(\mathbf{B})$. Em estudo epidemiológico, 
multicêntrico e prospectivo analisou-se uma amostra heterogênea de 265 pacientes. Observou-se que 85 pacientes (32,1\%) apresentavam síndrome compartimental abdominal (SCA) e a mortalidade foi significativamente maior entre estes indivíduos (38.8\% vs. $22.2 \%, p<0,005)$. Os fatores preditores de SCA foram: disfunção hepática (OR; 2,25; IC95\% 1,1-4,58; p < 0,03), cirurgia abdominal (OR: 1,96; IC95\% 1,05-3,64; $p<$ 0,03), ressuscitação volêmica (OR: 1,88; IC95\% 1,04-3,42; $\mathrm{p}<$

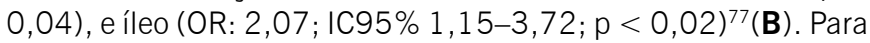
manter a meta de oferta tecidual de oxigênio adequada, a infusão de cristalóides durante as 24 horas iniciais tende a ser substancialmente maior ( 6 a 10 litros) do que quando a opção recai sobre a infusão de colóides (2 a 4 litros) $)^{2}(\mathbf{A})^{1}(\mathbf{B})^{29}(\mathbf{D})$. O estudo SAFE, ensaio clínico randomizado realizado em 16 hospitais da Oceania, incluiu 6997 pacientes que necessitaram de ressuscitação hídrica por depleção volêmica, e comparou os efeitos da ressuscitação hídrica realizada com albumina $4 \%$ e solução salina fisiológica sobre a mortalidade e morbidade. Embora os balanços hídricos das $24(p<0,001), 48(p<0,001)$ e 72 horas $(p=0,007)$ tenham sido significativamente maiores no grupo que recebeu cristalóides, não houve diferença na mortalidade após 28 dias de seguimento, permanência em ventilação mecânica, na UTI e hospitalar, bem como na duração de terapia renal substitutiva e no número de disfunções orgânica ${ }^{26}(\mathbf{A})$.

Recomendações: A expansão volêmica agressiva que resulta em maior balanço hídrico ao final das 1 as 6 horas de tratamento em busca da normalização de parâmetros hemodinâmicos como PAM, débito urinário e $\mathrm{ScvO} 2$, reduz a mortalidade da sepse grave e choque séptico²(A). A positivação tardia do balanço hídrico (expansão hídrica tardia) na sepse grave e choque séptico está associada a mais disfunções orgânicas e maior mortalidade $2(\mathbf{A})$. A expansão volêmica em busca da supranormalização dos parâmetros hemodinâmicos não deve ser utilizada por influenciar negativamente o prognóstico ${ }^{9}(\mathbf{A})$. O balanço hídrico acumulado ao final das primeiras 72 horas de tratamento não influencia a mortalidade e morbidade de populações heterogêneas ${ }^{26}(\mathbf{A})$. 0 excesso de oferta hídrica nas primeiras 72 horas está associado a piora da função pulmonar, maior permanência na ventilação mecânica, mais disfunções orgânicas e maior mortalidade em indivíduos com sepse e/ou SDRA/LPA ${ }^{73}(\mathbf{B})^{74}(\mathbf{A})$. Após o desenvolvimento da insuficiência renal em pacientes sépticos, a infusão hídrica adicional não se associa à recuperação da função renal podendo resultar em piora da função respiratória ${ }^{72}(\mathbf{B})$. 0 excesso de oferta hídrica pode resultar em síndrome compartimental abdominal em pacientes graves ${ }^{77}(\mathbf{B})$.

\section{Referências}

1. Vincent JL, Gerlach H. Fluid resuscitation in severe sepsis and septic shock: an evidence based review. Crit Care Med 2004;32(11 Suppl):S451-4.

2. Rivers E, Nguyen B, Havstad S, Ressler J, Muzzin A, Knoblich B, et al. Early goal-directed therapy in the treatment of severe sepsis and septic shock. N Engl J Med 2001;345:368-77.

3. Kern JW, Shoemaker WC. Meta-analysis of hemodynamic optimization in high-risk patients. Crit Care Med 2002;30:1686-92.

4. Dellinger RP, Levy MM, Carlet JM, Bion J, Parker MM, Jaeschke R, et al.
Surviving Sepsis Campaign: international guidelines for management of severe sepsis and septic shock: 2008. Crit Care Med 2008;36:296-327.

5. Otero RM, Nguyen HB, Huang DT, Gaieski DF, Goyal M, Gunnerson KJ, et al. Early goaldirected therapy in severe sepsis and septic shock revisited: concepts, controversies, and contemporary findings. Chest 2006;130:1579-95.

6. Rhodes A, Bennett ED. Early goal-directed therapy: an evidence-based review. Crit Care Med 2004;32(11 Suppl):S448-50.

7. Goldman RH, Klughaupt M, Metcalf T, Spivack AP, Harrison DC. Measurement of central venous oxygen saturation in patients with myocardial infarction. Circulation 1968;38:941-6.

8. Rady MY, Rivers EP, Nowak RM. Resuscitation of the critically ill in the ED: responses of blood pressure, heart rate, shock index, central venous oxygen saturation, and lactate. Am J Emerg Med 1996;14:218-25.

9. Gattinoni L, Brazzi L, Pelosi P, Latini R, Tognoni G, Pesenti A, et al. A trial of goal-oriented hemodynamic therapy in critically ill patients. SvO2 Collaborative Group. N Engl J Med 1995;333:1025-32.

10. Kumar A, Anel R, Bunnell E, Habet K, Zanotti S, Marshall S, et al. Pulmonary artery occlusion pressure and central venous pressure fail to predict ventricular filling volume, cardiac performance, or the response to volume infusion in normal subjects. Crit Care Med 2004;32:691-9.

11. Michard F, Boussat S, Chemla D, Anguel N, Mercat A, Lecarpentier Y, et al. Relation between respiratory changes in arterial pulse pressure and fluid responsiveness in septic patients with acute circulatory failure. Am J Respir Crit Care Med 2000;162:134-8.

12. Osman D, Ridel C, Ray P. Cardiac filling pressures are not appropriate to predict hemodynamic response to volume challenge. Crit Care Med 2007;35:64-8

13. Boldt J, Lenz M, Kumle B, Papsdorf M. Volume replacement strategies on intensive care units: results from a postal survey. Intensive Care Med 1998;24: 147-51.

14. Donnino MW, Clardy P, Talmor D. A central venous pressure goal of 8-12 $\mathrm{mm} \mathrm{Hg}$ for all patients in septic shock. Crit Care Med 2007;35:1441.

15. Hofer CK, Müller SM, Furrer L, Klaghofer R, Genoni M, Zollinger A. Stroke volume and pulse pressure variation for prediction of fl uid responsiveness in patients undergoing off - pump coronary artery bypass Graft ing. Chest 2005; 128:848-54.

16. Kramer A, Zygun D, Hawes H, Easton P, Ferland A. Pulse pressure variation predicts fluid responsiveness following coronary artery bypass surgery. Chest 2004;126:1563-8.

17. Feissel M, Teboul JL, Merlani P, Badie J, Faller JP, Bendjelid K. Plethysmographic dynamic indices predict fluid responsiveness in septic ventilated patients. Intensive Care Med 2007;33:993-9.

18. Wyff els PAH, Durnez PJD, Helderweirt J, Stockman WMA, De Kegel $D$. Ventilation induced plethysmographic variations predict fluid responsiveness in ventilated postoperative cardiac surgery patients. Anesth Analg 2007; 105:448-52.

19. Natalini G, Rosano A, Taranto M, Faggian B, Vitorielli E, Bernardini A. Arterial versus plethysmographic dynamic indices to test responsiveness for testing fluid administration in hypotensive patients: A clinical trial. Anesth Analg 2006;103:1478-84.

20. Magder SA, Georgiadis G, Cheong T. Respiratory variations in right atrial pressure predict response to fluid challenge. J Crit Care 1992;7:76-85.

21. Magder S, Lagonidis D. Eff ectiveness of albumin versus normal saline as a test of volume responsiveness in post-cardiac surgery patients. J Crit Care 1999;14:164-71.

22. Heenen S, De Backer D, Vincent JL. How can the response to volume expansion in patients with spontaneous respiratory movements be predicted? Critical Care 2006 acesso em 02 mai. 2009;10:R102. Disponível em: http:// ccforum.com/content/10/4/R102

23. Cochrane Injuries Group Albumin Reviewers. Human albumin administration in critically ill patients: systematic review of randomised controlled trials. BMJ 1998;317:235-40.

24. Schierhout G, Roberts I. Fluid resuscitation with colloid or crystalloid. BM 1998;316:961-4.

25. Perel P, Roberts I. Colloids versus crystalloids for fluid resuscitation in 
critically ill patients. Cochrane Database Syst Rev 2007;(4):CD000567. 26. Finfer S, Bellomo R, Boyce N, French J, Myburgh J, Norton R; SAFE Study Investigators. A comparison of albumin and saline for fl uid resuscitation in the intensive care unit. N Engl J Med 2004;350:2247-56.

27. SAFE Study Investigators, Australian and New Zealand Intensive Care Society Clinical Trials Group, Australian Red Cross Blood Service, George Institute for International Health, Myburgh J, Cooper DJ, et al. Saline or albumin for fluid resuscitation in patients with traumatic brain injury. N Engl J Med 2007;357:874-84

28. SAFE Study Investigators, Finfer S, Bellomo R, McEvoy S, Lo SK, Myburgh $\mathrm{J}$, et al. Effect of baseline serum albumin concentration on outcome of resuscitation with albumin or saline in patients in intensive care units: analysis of data from the saline versus albumin fluid evaluation (SAFE) study. BMJ 2006; 333:1044-50.

29. Hollenberg SM, Ahrens TS, Annane D, Astiz ME, Chalfi n DB, Dasta JF, et al. Practice parameters for hemodynamic support of sepsis in adult patients: 2004 update. Crit Care Med 2004;32:1928-48.

30. Martin C, Papazian L, Perrin G, Saux P, Gouin F. Norepinephrine or dopamine for the treatment of hyperdynamic septic shock. Chest 1993;103:1826-31. 31. Levy B, Bollaert PE, Charpentier C, Nace L, Audibert G, Bauer P, et al. Comparison of norepinephrine and dobutamine to epinephrine for hemodynamics, lactate metabolism, and gastric tonometric variables in septic shock: a prospective, randomized study. Intensive Care Med 1997;23:282-7.

32. Meier-Hellmann A, Bredle DL, Specht M, Spies C, Hannemann L, Reinhart $K$. The effects of low-dose dopamine on splanchnic blood flow and oxygen utilization in patients with septic shock. Intensive Care Med 1997;23:31-7. 33. Wilson RF, Sibbald WJ, Jaanimagi JL. Hemodynamic eff ects of dopamine in critically ill septic patients. J Surg Res 1976;20:163-72.

34. Schreuder WO, Schneider AJ, Groeneveld AB, Thijs LG. Effect of dopamine vs norepinephrine on hemodynamics in septic shock. Emphasis on right ventricular performance. Chest 1989;95:1282-8.

35. Beale RJ, Hollenberg SM, Vincent JL, Parrillo JE. Vasopressor and inotropic support in septic shock: an evidence based review. Crit Care Med 2004;32:S455-65.

36. Ruokonen E, Takala J, Kari A, Saxén H, Mertsola J, Hansen EJ. Regional blood flow and oxygen transport in septic shock. Crit Care Med 1993;21:1296-303.

37. Marik PE, Mohedin M. The contrasting eff ects of dopamine and norepinephrine on systemic and splanchnic oxygen utilization in hyperdynamic sepsis. JAMA 1994;272:1354-7.

38. De Backer D, Creteur J, Silva E, Vincent JL. Effects of dopamine, norepinephrine, and epinephrine on the splanchnic circulation in septic shock: which is best? Crit Care Med 2003;31:1659-67.

39. Bellomo R, Chapman M, Finfer S, Hickling K, Myburgh J. Low-dose dopamine in patients with early renal dysfunction: a placebo-controlled randomised trial. Australian and New Zealand Intensive Care Society (ANZICS) Clinical Trials Group. Lancet 2000;356:2139-43.

40. Desjars P, Pinaud M, Bugnon D, Tasseau F. Norepinephrine therapy has no deleterious renal effects in human septic shock. Crit Care Med 1989;17:426-9.

41. Redl-Wenzl EM, Armbruster C, Edelmann G, Fischl E, Kolacny M, WechslerFördös $A$, et al. The effects of norepinephrine on hemodynamics and renal function in severe septic shock states. Intensive Care Med 1993;19:151-4. 42. Marin C, Eon B, Saux P, Aknin P, Gouin F. Renal effects of norepinephrine used to treat septic shock patients. Crit Care Med 1990;18:282-5.

43. Sakr Y, Reinhart K, Vincent JL, Sprung CL, Moreno R, Ranieri VM, et al. Does dopamine administration in shock influence outcome? Results of the Sepsis Occurrence in Acutely III Patients (SOAP) Study. Crit Care Med 2006; 34:589-97.

44. Day NP, Phu NH, Bethell DP, Mai NT, Chau TT, Hien TT, et al. The effects of dopamine and adrenaline infusions on acid-base balance and systemic haemodynamics in severe infection. Lancet 1996;348:219-23.

45. Sharshar T, Blanchard A, Paillard M, et al. Circulating vasopressin levels in septic shock. Crit Care Med 2003;31:1752-8.

46. Sharshar T, Carlier R, Blanchard A, Feydy A, Gray F, Paillard M, et al.
Depletion of neurohypophyseal content of vasopressin in septic shock. Crit Care Med 2002;30:497-500.

47. Holmes CL, Walley KR, Chittock DR, Lehman T, Russell JA. The effects of vasopressin on hemodynamics and renal function in severe septic shock: a case series. Intensive Care Med 2001;27:1416-21.

48. Landry DW, Levin HR, Gallant EM, Ashton RC Jr, Seo S, D'Alessandro D, et al. Vasopressin deficiency contributes to the vasodilation of septic shock. Circulation 1997;95:1122-5.

49. Malay MB, Ashton RC Jr, Landry DW, Townsend RN., et al. Lowdose vasopressin in the treatment of vasodilatory septic shock. J Trauma 1999;47:699-703.

50. Patel BM, Chittock DR, Russell JA, Walley KR. Beneficial effects of short-term vasopressin infusion during severe septic shock. Anesthesiology 2002;96:576-8.

51. van Haren FM, Rozendaal FW, van der Hoeven JG. The effect of vasopressin on gastric perfusion in catecholamine-dependent patients in septic shock. Chest 2003;124:2256-60.

52. Klinzing S, Simon M, Reinhart K, Bredle DL, Meier-Hellmann A., et al. High-dose vasopressin is not superior to norepinephrine in septic shock. Crit Care Med 2003;31:2646-50.

53. Russell JA, Walley KR, Singer J, Gordon AC, Hébert PC, Cooper DJ, et al. Vasopressin versus norepinephrine infusion in patients with septic shock. $N$ Engl J Med 2008;358:877-87.

54. Martin C, Papazian L, Perrin G, Saux P, Gouin F. Norepinephrine or dopamine for the treatment of hyperdynamic septic shock. Chest 1993;103:1826-31. 55. Levy B, Nace L, Bollaert PE, Dousset B, Mallie JP, Larcan A. Comparison of systemic and regional effects of dobutamine and dopexamine in norepinephrine-treated septic shock. Intensive Care Med 1999;25:942-8.

56. Meier-Hellmann A, Reinhart K, Bredle DL, Specht M, Spies CD, Hannemann L. Epinephrine impairs splanchnic perfusion in septic shock. Crit Care Med 1997;25:399-404

57. Barton P, Garcia J, Kouatli A, Kitchen L, Zorka A, Lindsay C, et al. Hemodynamic effects of i.v. milrinone lactate in pediatric patients with septic shock. A prospective, double-blinded, randomized, placebo-controlled, interventional study. Chest 1996;109:1302-12.

58. Hernández G, Gigoux J, Bugedo G, Castillo L, Bruhn A, Tomicic V, et al. Acute effect of dobutamine and amrinone on hemodynamics and splanchnic perfusion in septic shock patients. Rev Med Chil 1999;127:660-6.

59. Heinz G, Geppert A, Delle Karth G, Reinelt P, Gschwandtner ME, Neunteufl $\mathrm{T}$, et al. IV milrinone for cardiac output increase and maintenance: comparison in nonhyperdynamic SIRS/sepsis and congestive heart failure. Intensive Care Med 1999;25:620-4.

60. Loeb HS, Winslow EB, Rahimtoola SH, Rosen KM, Gunnar RM. Acute hemodynamic effects of dopamine in patients with shock. Circulation 1971;44:163-73.

61. Winslow EJ, Loeb HS, Rahimtoola SH, Kamath S, Gunnar RM. Hemodynamic studies and results of therapy in 50 patients with bacteremic shock. Am J Med 1973;54:421-32.

62. Packer M. REVIVE II: multicenter placebo-controlled trial of levosimendan on clinical status in acutely decompensated heart failure. In: Program and abstracts from the American Heart Association Scientific Sessions; 2005 Nov 13-16; Dallas, Texas. Dallas: American Heart Association, [2005].

63. Mebazaa A. The SURVIVE-W Trial: comparison of dobutamine and levosimendan on survival in acute decompensated heart failure. In: Program and abstracts from the American Heart Association Scientific Sessions; 2005 Nov 13-16; Dallas, Texas. Dallas: American Heart Association, [2005].

64. Cooper DJ, Wallwy KR, Wiggs BR, Russel JA. Bicarbonate does not improve hemodynamics in critically ill patients who have lactic acidosis. A prospective, controlled clinical study. Ann Intern Med 1990;112:492-8.

65. Mathieu D, Neviere R, Billard V, Fleyfel M, Wattel F. Effects of bicarbonate therapy on hemodynamics and tissue oxygenation in patients with lactic acidosis: a prospective, controlled clinical study. Crit Care Med 1991;19:1352-6. 66. Viallon A, Zeni F, Lafond $P$, et al. Does bicarbonate therapy improve the management of severe diabetic ketoacidosis? Crit Care Med 1999;27:2690-3. 67. Bing $\mathrm{OH}$, Brooks WW, Messer JV. Heart muscle viability following hypoxia: 
protective effect of acidosis. Science 1973;180:1297-8.

68. Morimoto Y, Morimoto Y, Kemmotsu O, Alojado ES. Extracellular acidosis delays cell death against glucose-oxygen deprivation in neuroblastoma $x$ glioma hybrid cells. Crit Care Med 1997;25:841-7.

69. Unno N, Menconi MJ, Smith M, Hagen SJ, Brown DA, Aguirre DE, et al. Acidic conditions ameliorate both adenosine triphosphate depletion and the development of hyperpermeability in cultured Caco-2BBe enterocytic monolayers subjected to metabolic inhibition. Surgery 1997;121:668-80.

70. Hayes MA, Timmins AC, Yau EHS, Palazzo M, Hinds CJ, Watson D. Elevation of systemic oxygen delivery in the treatment of critically ill patients. $\mathrm{N}$ Engl J Med 1994;-330:1717-22.

71. Michard F, Teboul JL. Predicting fluid responsiveness in ICU patients: a critical analysis of the evidence. Chest 2002;121:2000-8.

72. Van Biesen W, Yegenaga I, Vanholder R, Verbeke F, Hoste E, Colardyn F, et al. Relationship between fluid status and its management on acute renal failure (ARF) in intensive care unit (ICU) patients with sepsis: a prospective analysis. J Nephrol 2005;18:54-60.

73. Sakr Y, Vincent JL, Reinhart K, Groeneveld J, Michalopoulos A, Sprung CL, et al. High tidal volume and positive fluid balance are associated with worse outcome in acute lung injury. Chest 2005;128:3098-108.

74. National Heart, Lung, and Blood Institute Acute Respiratory Distress Syndrome (ARDS) Clinical Trials Network, Wiedemann HP, Wheeler AP, Bernard GR, Thompson BT, Hayden D, deBoisblanc B, et al. Comparison of two fluid management strategies in acute lung injury. N Engl J Med 2006;354:2564-75. 75. Alsous F, Khamiees M, DeGirolamo A, Amoateng-Adjepong Y, Manthous CA. Negative fluid balance predicts survival in patients with septic shock: a retrospective pilot study. Chest 2000;117:1749-54.

76. Vincent JL, Sakr Y, Sprung CL, Ranieri VM, Reinhart K, Gerlach H, et al. Sepsis in European intensive care units: results of the SOAP study. Crit Care Med 2006;34:344-53.

77. Malbrain MLNG, Chiumello D, Pelosi P, Bihari D, Innes R, Ranieri VM, et al. Incidence and prognosis of intraabdominal hypertension in a mixed population of critically ill patients: a multicenter epidemiological study. Crit Care Med 2005; 33:315-22. 